\title{
Partial sensitization of human bladder cancer cells to a gene-therapeutic adenovirus carrying REIC/Dkk-3 by downregulation of BRPK/PINK1
}

\author{
YU JIN $^{1}$, HITOSHI MURATA ${ }^{1}$, MASAKIYO SAKAGUCHI ${ }^{1}$, KEN KATAOKA ${ }^{1}$, \\ MASAMI WATANABE ${ }^{2}$, YASUTOMO NASU ${ }^{2}$, HIROMI KUMON ${ }^{2}$ and NAM-HO HUH ${ }^{1}$ \\ Departments of ${ }^{1}$ Cell Biology and ${ }^{2}$ Urology, Okayama University Graduate School of Medicine, \\ Dentistry and Pharmaceutical Sciences, Kita-ku, Okayama 700-8558, Japan
}

Received August 31, 2011; Accepted October 18, 2011

DOI: $10.3892 /$ or.2011.1543

\begin{abstract}
REIC/Dkk-3 is a tumor suppressor gene that was first identified as a gene downregulated in association with immortalization of normal human fibroblasts. We have demonstrated that an adenovirus carrying REIC/Dkk-3 (Ad-REIC) showed a tumor-specific killing effect on a wide range of cancers. However, some human cancers, bladder cancers in particular, are resistant to Ad-REIC. In this study, we investigated the combination effect of downregulation of BRPK/PINK1 (PINK1) and Ad-REIC on bladder cancer cells. Five bladder cancer cell lines among six cell lines examined were resistant to Ad-REIC. Among the cell lines, the resistance of two cell lines was probably due to low infection efficiency of the adenovirus. PINK1-specific siRNA remarkably downregulated $\mathrm{Bcl}-\mathrm{x}_{\mathrm{L}}$ and TRAP1 proteins and upregulated BAX protein expression. Finally, downregulation of PINK1 partially sensitized the other three cell lines that were resistant to Ad-REIC. This sensitization was associated with increasing production of reactive oxygen species (ROS). These results indicate that PINK1 is one of the key molecules for the mitochondrial protection system and that PINK1 can be a new target molecule to sensitize bladder cancer cells that are resistant to Ad-REIC.
\end{abstract}

\section{Introduction}

REIC/Dkk-3 is a tumor suppressor gene that was first identified as a gene downregulated in association with immortalization of normal human fibroblasts (1). Subsequently, we found that overexpression of REIC/Dkk-3 using an adenovirus vector

Correspondence to: Dr Nam-Ho Huh, Department of Cell Biology, Graduate School of Medicine, Dentistry and Pharmaceutical Sciences, 2-5-1 Shikata-cho, Kita-ku, Okayama 700-8558, Japan E-mail:namu@md.okayama-u.ac.jp

Key words: REIC/Dkk-3, gene therapy, bladder cancer, BRPK/ PINK1, sensitization
(Ad-REIC) showed a tumor-specific killing effect on a wide range of cancers, including those derived from the prostate, testis, pleura, breast and stomach (2-6). However, some human cancers, bladder cancers in particular, are resistant to Ad-REIC partly because of high expression levels of mitochondrial antiapoptotic proteins such as Bcl-2 and Bcl- $\mathrm{x}_{\mathrm{L}}$ (7). Upon initiation of apoptotic signaling, the pro-apoptotic Bcl-2 protein BAX undergoes a conformation shift and is inserted into the outer mitochondrial membrane, which increases membrane permeability $(8,9)$. This results in the release of cytochrome $\mathrm{c}$ and other pro-apoptotic factors from the mitochondria, eventually leading to apoptosis. In contrast, $\mathrm{Bcl}-2$ and $\mathrm{Bcl}-\mathrm{x}_{\mathrm{L}}$ are antiapoptotic proteins and inhibit the release of pro-apoptotic factors from the mitochondria (10). Thus, the ratio between pro- and anti-apoptotic proteins of the Bcl-2 family is one of the critical determining factors for apoptosis, and such proteins function on the mitochondria.

The BRPK/PINK1 (PINK1) gene encodes a serine/ threonine kinase with a mitochondrial localization signal, and mutations of the gene are associated with autosomal recessive inheritance of Parkinson's disease (11). Overexpression of wild-type PINK1 protected neuronal cells against various stresses (12), whereas downregulation of PINK1 sensitized neuroblastoma cells to various stresses (13). On the other hand, we showed that PINK1 was expressed at high levels in malignant cancer cells exhibiting an increased metastatic activity (14) and that PINK1 protected cancer cells against various cytotoxic agents through Akt activation $(15,16)$. Martin et al (17) reported that PINK1 is a potential therapeutic target for the treatment of DNA mismatch repair-deficient cancers. Thus, accumulating lines of evidence indicate that PINK1 protects cancer cells from stress-mediated mitochondrial dysfunction, various stresses and apoptosis. In the present study, we examined the possibility that downregulation of PINK1 may sensitize bladder cancer cells that are resistant to Ad-REIC through inducing mitochondrial dysfunction.

\section{Materials and methods}

Cell culture. Human bladder cancer cell lines T24, J82, 5637, UM-UC-3 and TCCSUP were purchased from ATCC 
(Rockville, MD). The human bladder cancer cell line KK47 (18) was a gift from Dr Kumazawa (Department of Urology, Faculty of Medicine, Kyushu University). These cells were cultured in DMEM/F12 (Ham) (1:1) (Invitrogen, Carlsbad, CA) supplemented with $10 \%$ fetal bovine serum.

Adenovirus vectors and infection. Ad-REIC was produced and propagated as previously described (2). An adenovirus vector carrying the LacZ gene (Ad-LacZ) was used for monitoring infection efficiency. Twenty-four hours after subculture, cells were treated with Ad-LacZ or Ad-REIC at the indicated multiplicity of infection (MOI).

Apoptosis assay. Apoptotic cells were identified after staining with $0.2 \mu \mathrm{M}$ Hoechst 33342 (Invitrogen) for $30 \mathrm{~min}$. Under a fluorescence microscope, cells with fragmented or shrunk nuclei were counted as apoptotic cells.

Assay for adenovirus infection efficiency. To determine adenovirus infection efficiency, cells were infected with Ad-LacZ. Forty-eight hours after infection, the cells were washed with PBS, fixed at $4^{\circ} \mathrm{C}$ for 10 min with $0.25 \%$ glutar aldehyde, rinsed 4 times with PBS, and then stained with $2.5 \mathrm{mM}$ 5-bromo4-chloro-3-indolyl-D-galactoside (X-Gal) at $37^{\circ} \mathrm{C}$ for $18 \mathrm{~h}$.

Western blot analysis. Western blot analysis was performed under conventional conditions after lysing cells with M-PER mammalian protein extraction reagent (Thermo Scientific) with PhosSTOP Phosphatase Inhibitor (Roche Applied Science). The antibodies used were as follows: antibody against PINK1 (Novus); antibodies against BiP, TRAP1, Bcl- $\mathrm{X}_{\mathrm{L}}$, and BAX (BD Pharmingen, San Jose, CA); antibodies against Grp75 and Bcl-2 (Santa Cruz Biotechnology, Santa Cruz, CA); antibody against $\beta$-actin (Sigma); and HRP-labeled anti-mouse and anti-rabbit secondary antibodies (Cell Signaling Technology, Danvers, MA).

$R T-P C R$. Total RNA was extracted with the SV Total RNA Isolation system (Promega, Madison, WI). The Total RNA was used to synthesize cDNA with the Superscript III FirstStrand Synthesis system (Invitrogen). RT-PCR was performed under conventional conditions. The primers used were as follows: human PINK1 (forward) 5'-CACCTTGAAAGCC GCAGCTACCAAGA-3', human PINK1 (reverse) 5'-AGC

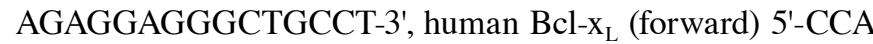

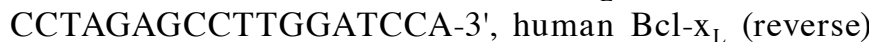
5'-ACGCCGGCCACAGTCATG-3', human Bcl-2 (forward) 5'-CCCTGGTGGACAACATCGC-3', human Bcl-2 (reverse) 5'-CCAGGAGAAATCAAACAGAGGC-3', human glyceraldehyde-3-phosphate dehydrogenase (GAPDH: forward) 5'-ATTCCATGGCACCGTCAAGGCT-3', human GAPDH (reverse) 5'-TCAGGTCCACCACTGACACGTT-3'.

RNA interference. siGENOME SMARTpool siRNA targeting PINK1 (NM_032409) (Thermo Scientific Dharmacon, Lafayette, $\mathrm{CO}$ ) was transfected into cells using Lipofectamine RNAi MAX (Invitrogen). A control siRNA with no known mammalian homology (siGENOME non-targeting siRNA pool 1, Thermo Scientific Dharmacon) was used as a negative control.
A

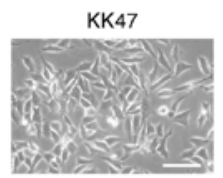

UM-UC-3

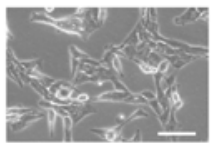

T24

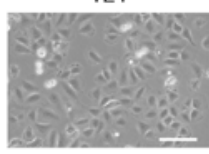

$\mathrm{J} 82$
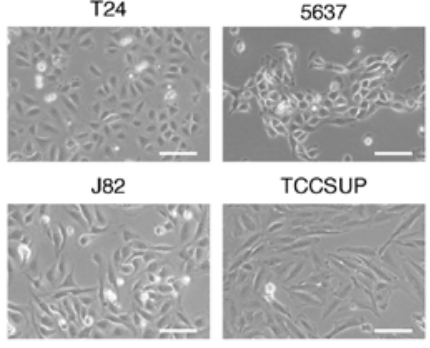

TCCSUP

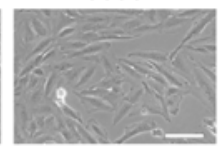

B
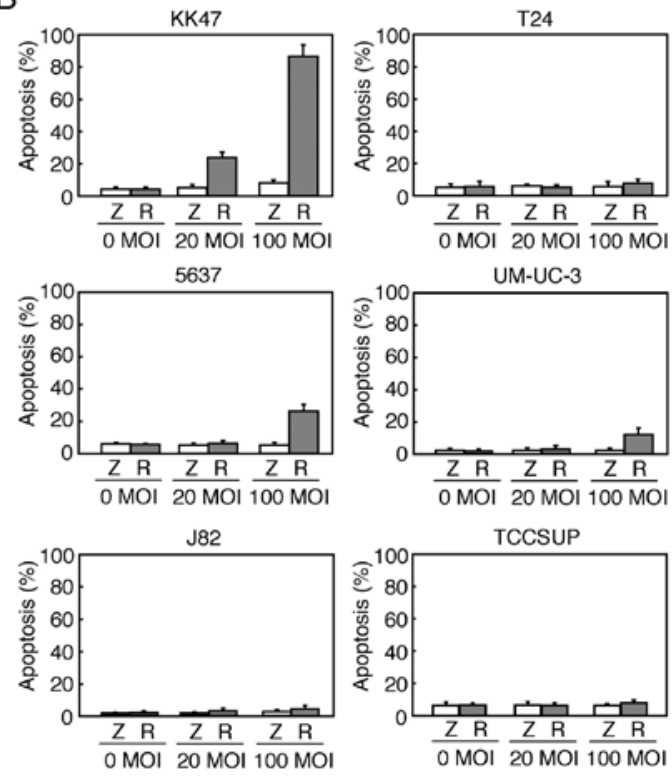

Figure 1. Resistance of bladder cancer cell lines to Ad-REIC-induced apoptosis. (A) Morphology of human bladder cancer cell lines. Bars, $100 \mu \mathrm{m}$. (B) Rates of apoptosis induction by Ad-REIC in bladder cancer cell lines. Cells were infected with an adenovirus vector carrying REIC (R) or LacZ (Z). Fourty-eight hours after the infection, apoptotic cells were determined by staining with Hoechst 33342.

Assay for reactive oxygen species (ROS) formation. To visualize intracellular ROS, BES- $\mathrm{H}_{2} \mathrm{O}_{2}$ staining was performed. Cells were incubated with $1 \mu \mathrm{M}$ BES- $\mathrm{H}_{2} \mathrm{O}_{2}$ (Wako Chemicals, Osaka, Japan) at $37^{\circ} \mathrm{C}$ for $1 \mathrm{~h}$. After washout by rinsing thoroughly, BES- $\mathrm{H}_{2} \mathrm{O}_{2}$ signal was detected by $488 \mathrm{~nm}$ excitation.

\section{Results}

Resistance of human bladder cancer cell lines to Ad-REICinduced apoptosis. At first, we examined the sensitivity to Ad-REIC using 6 bladder cancer cell lines, KK47, T24, 5637, UM-UC-3, J82 and TCCSUP (Fig. 1A). Among those cell lines, only KK47 was sensitive to the induction of apoptosis by Ad-REIC, and the other 5 cell lines underwent apoptosis at marginal rates even at $100 \mathrm{MOI}$ (Fig. 1B).

To reveal underlying mechanisms of the difference in the sensitivities of the two groups, we first examined the infection efficiency of an adenovirus. The infection efficiency of Ad-LacZ of KK47, T24, 5637 and UM-UC-3 was high, but J82 and TCCSUP showed lower infection efficiency (Fig. 2). Since our previous studies showed that BiP/Grp78 and the Bcl-2 family proteins are responsible for resistance to Ad-REIC $(7,20)$, we examined the expression levels of heat shock/ 
A

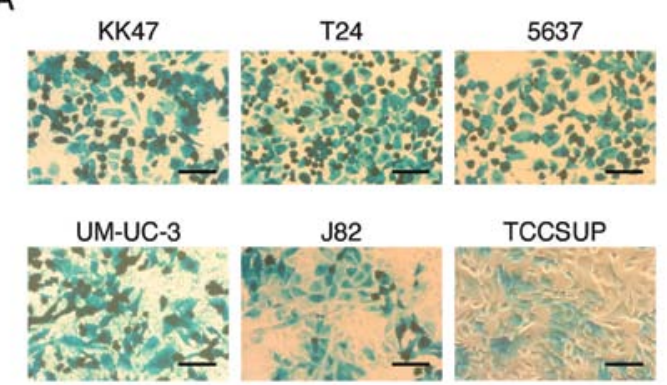

B

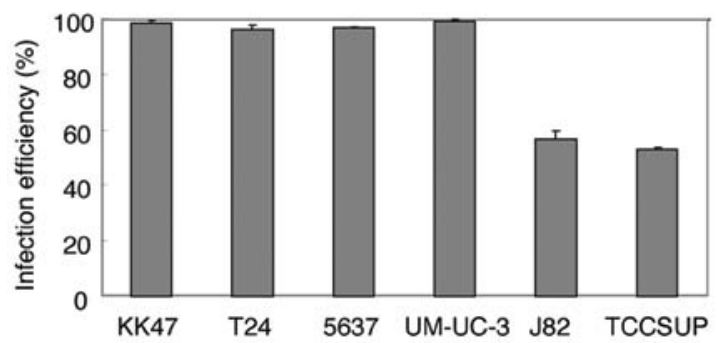

Figure 2. Infection efficiency of adenovirus in bladder cancer cell lines. (A) Cells were infected with an adenovirus vector carrying LacZ (100 MOI) After $48 \mathrm{~h}, \beta$-galactosidase activity was visualized by staining with X-gal. Bars, $100 \mu \mathrm{m}$. (B) Rate of infected cells in bladder cancer cell lines.

chaperone proteins and $\mathrm{Bcl}-2$ family proteins (Fig. 3). No significant change in the expression of BiP, Grp75 and TRAP1 was observed in the bladder cancer cell lines. On the other hand, $\mathrm{Bcl}-\mathrm{x}_{\mathrm{L}}$ and $\mathrm{Bcl}-2$ were upregulated in Ad-REIC-resistant cancer cell lines. These results suggest that the resistance of bladder cancer cells to Ad-REIC was partly due to low infection efficiency of Ad-REIC (J82 and TCCSUP) and high expression of mitochondrial anti-apoptotic proteins such as Bcl- $\mathrm{x}_{\mathrm{L}}$ and Bcl-2 (T24, 5637, UM-UC-3, J82 and TCCSUP).

Protein expression of $B c l-x_{L}$ is reduced by downregulation of PINK1. Since our previous studies showed that PINK1, a mitochondrial protein, could protect cancer cells, we explored the use of PINK1 as a target to overcome the resistance of bladder cancer cells to Ad-REIC-induced apoptosis. Downregulation of endogenous PINK1 by siRNA reduced basal levels of TRAP1 and Bcl- $\mathrm{x}_{\mathrm{L}}$ and increased basal level of BAX in T24 cells (Fig. 4A). RT-PCR analysis revealed that mRNA expression level of $\mathrm{Bcl}-\mathrm{x}_{\mathrm{L}}$ was not changed by downregulation of PINK1 (Fig. 4B).

Downregulation of PINK1 sensitizes bladder cancer cells that are resistant to Ad-REIC-induced apoptosis. We examined the combination effect of downregulation of PINK1 and Ad-REIC for apoptosis induction. We used T24, 5637 and UM-UC-3 cells because these cell lines are resistant to Ad-REIC by high expression of mitochondrial anti-apoptotic proteins but not by low infection efficiency of adenovirus. Overexpression of REIC/Dkk-3 following downregulation of PINK1 induced apoptosis at significantly higher levels in all three cell lines (Fig. 5).

Downregulation of PINK1 augments MG132-induced ROS formation. Cellular level of hydrogen peroxide, a major ROS,

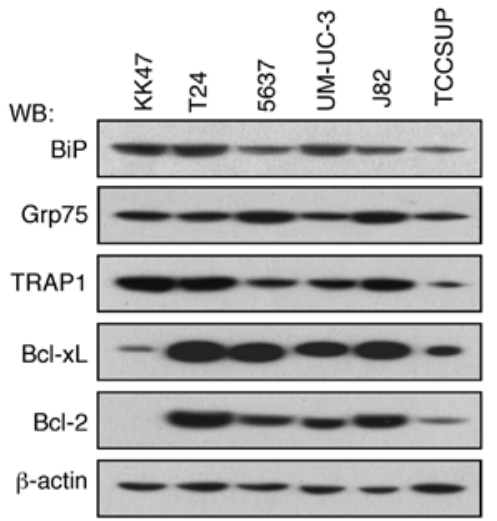

Figure 3. Expression of heat shock/chaperone proteins and Bcl-2 family proteins in bladder cancer cell lines. Western blot analysis (WB) for heat shock/ chaperone proteins and $\mathrm{Bcl}-2$ family proteins was performed. $\beta$-actin was used as a loading control.
A

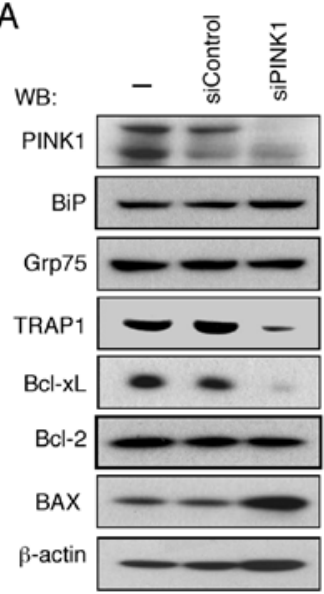

B

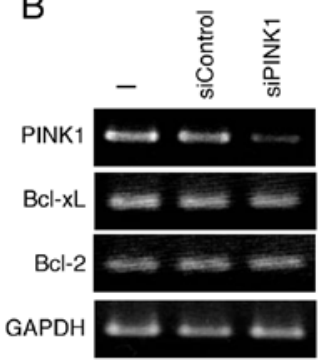

Figure 4. Effect of downregulation of PINK1. (A) Western blot analysis (WB) for heat shock/chaperone proteins and Bcl-2 family proteins was performed $72 \mathrm{~h}$ after transfection of siRNA for PINK1. (B) The relative mRNA levels of Bcl- $\mathrm{x}_{\mathrm{L}}$ and Bcl-2 were measured by RT-PCR. GAPDH was used as a loading control.

was determined by BES- $\mathrm{H}_{2} \mathrm{O}_{2}$ staining. Hydrogen peroxide produced by stimulation with MG-132 was significantly increased in PINK1-downregulated cells compared with that in untreated cells (Fig. 6).

\section{Discussion}

In this study, we showed that downregulation of PINK1 partially sensitized bladder cancer cells to Ad-REIC in vitro. As shown in Fig. 1, five bladder cancer cell lines (T24, 5637, UM-UC-3, J82 and TCCSUP) were resistant and only one cell line (KK47) was sensitive to Ad-REIC treatment. Infection efficiency of the adenovirus of two cell lines (J82 and TCCSUP) was limited and this may explain, at least partly, the lower sensitivity to Ad-REIC. A possibility to overcome this obstacle is to create a new version of the adenovirus vector that has higher infection efficiency and potent gene expression capacity. This project has almost been completed in our laboratory.

Our previous study revealed that the Bcl-2 family of mitochondrial proteins was responsible for resistance of bladder 

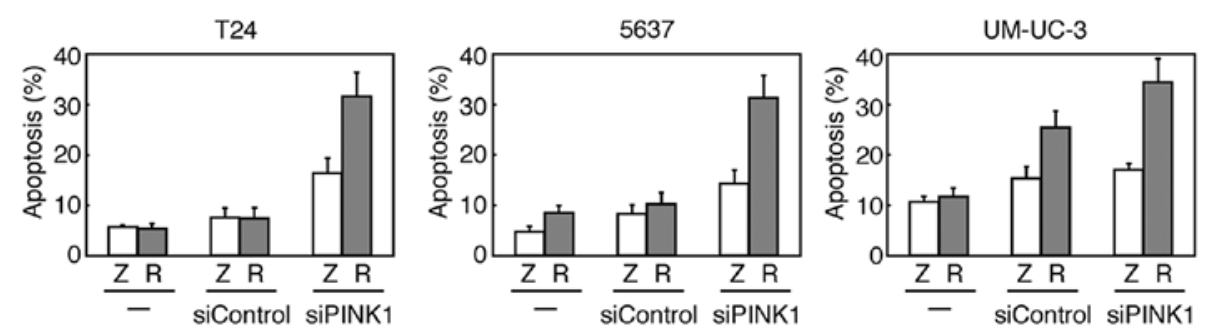

Figure 5. Sensitization of bladder cancer cells to Ad-REIC by downregulation of PINK1. T24, 5637 and UM-UC-3 cells were transfected with indicated siRNAs. Forty-eight hours later, cells were infected with an adenovirus vector (100 MOI) carrying REIC (R) or LacZ (Z), and apoptotic rate of cells was determined after incubating for another $48 \mathrm{~h}$ by staining with Hoechst 33342.

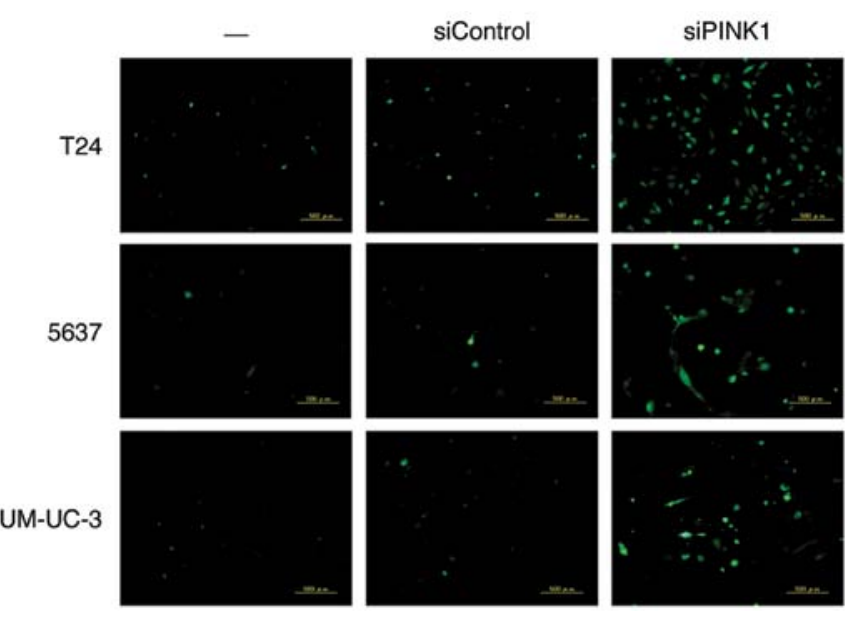

Figure 6. Downregulation of PINK1 augments MG132-induced ROS formation. T24, 5637 and UM-UC-3 cells were transfected with indicated siRNAs $60 \mathrm{~h}$ prior to treatment with MG-132 $(1 \mu \mathrm{M}, 12 \mathrm{~h})$. BES- $\mathrm{H}_{2} \mathrm{O}_{2}$ staining $(1 \mu \mathrm{M}$, $1 \mathrm{~h}$ ) was performed to visualize hydrogen peroxide. Bars, $500 \mu \mathrm{m}$.

cancer cells to Ad-REIC (7). In accordance with this, Bcl-2 family proteins were upregulated in all resistant cell lines (Fig. 3). One possibility to sensitize these cell lines to Ad-REIC is to apply Bcl-2 inhibitors, but our previous study showed that typical Bcl-2 inhibitors were non-selective and showed strong toxicity to normal cells as well as cancer cells (19).

We also reported that resistant clones isolated from sesitive PC3 prostate cancer cells expressed BiP/Grp78, an ER-resisting chaperone protein, at enhanced levels (20). Downregulation of BiP/Grp78 with specific siRNA sensitized the resistant clones to Ad-REIC. Scirrhous gastric cancer cells were shown to be resistant to Ad-REIC and to express BiP/ Grp78 at a higher level (data not shown). On the other hand, levels of BiP/Grp78, Grp75 and TRAP1 in the resistant bladder cancer cell lines were not as high as those in scirrhous gastric cancer cells, and no remarkable difference in the expression levels of BiP/Grp78, Grp75 and TRAP1 was noted between the sensitive and resistant bladder cancer cell lines (Fig. 3). These results indicate that the strategy using $\mathrm{BiP} / \mathrm{Grp} 78$ inhibition is not promising for sensitizing Ad-REIC-resistant cancer cells.

Mitochondria are dynamic organelles that generate energy for cell functions and regulate apoptosis. Recent studies have revealed that interaction of mitochondria and ER contributes to induction of apoptosis (21). For example, apoptosis signaling from the ER is passed to mitochondria. Recently, Iwasawa et al revealed that mitochondria transmitted signals to the ER to regulate apoptosis (22). Our previous research demonstrated that Ad-REIC induced apoptosis through ER stress (23). Thus, mitochondria are key organelles through which modulation of cellular sensitivity to Ad-REIC could be developed.

To regulate mitochondria function, we focused on PINK1, a familial Parkinson's disease-linked gene. Downregulation of BRPK/PINK1 has been previously reported to sensitize neuroblastoma cells to various stresses (13). Our hypothetic strategy for sensitizing resistant bladder cancer cells to Ad-REIC was mitochondria dysfunction induced by downregulation of PINK1. Indeed, downregulation of PINK1 could sensitize resistant bladder cancer cells to Ad-REIC (Fig. 5). Furthermore, our preliminary experiments demonstrate that downregulation of PINK1 in resistant bladder cancer cells under similar conditions resulted in sensitization of the cells to oxidative stress induced by MG-132 and enhanced production of ROS (Fig. 6). The results of the present and previous studies (15) indicate that PINK1 can be a new target molecule to sensitize resistant bladder cancer cells to Ad-REIC.

\section{Acknowledgements}

This study was supported in part by grants from the Ministry of Education, Culture, Sports, Science, and Technology of Japan (23650625 to N.-H.H. and 21591699 to K.K.).

\section{References}

1. Tsuji T, Miyazaki M, Sakaguchi M, Inoue $\mathrm{Y}$ and Namba M: A REIC gene shows down-regulation in human immortalized cells and human tumor-derived cell lines. Biochem Biophys Res Commun 268: 20-24, 2000.

2. Abarzua F, Sakaguchi M, Takaishi M, Nasu Y, Kurose K, Ebara S, Miyazaki M, Namba M, Kumon H and Huh NH: Adenovirusmediated overexpression of REIC/Dkk-3 selectively induces apoptosis in human prostate cancer cells through activation of c-Jun- $\mathrm{NH}_{2}$-kinase. Cancer Res 65: 9617-9622, 2005.

3. Tanimoto R, Abarzua F, Sakaguchi M, Takaishi M, Nasu Y, Kumon $\mathrm{H}$ and Huh NH: REIC/Dkk-3 as a potential gene therapeutic agent against human testicular cancer. Int J Mol Med 19: 363-368, 2007.

4. Kashiwakura Y, Ochiai K, Watanabe M, Abarzua F, Sakaguchi M, Takaoka M, Tanimoto R, Nasu Y, Huh NH and Kumon H: Down-regulation of inhibition of differentiation-1 via activation of activating transcription factor 3 and Smad regulates REIC/Dickkopf-3-induced apoptosis. Cancer Res 68: 8333-8341, 2008.

5. Kawasaki K, Watanabe M, Sakaguchi M, Ogasawara Y, Ochiai K, Nasu Y, Doihara H, Kashiwakura Y, Huh NH, Kumon H and Date H: REIC/Dkk-3 overexpression downregulates P-glycoprotein in multidrug-resistant MCF7/ADR cells and induces apoptosis in breast cancer. Cancer Gene Ther 16: 65-72, 2009. 
6. Than SS, Kataoka K, Sakaguchi M, Murata H, Abarzua F Taketa C, Du G, Yashiro M, Yanagihara K, Nasu Y, Kumon H and Huh NH: Intraperitoneal administration of an adenovirus vector carrying REIC/Dkk-3 suppresses peritoneal dissemination of scirrhous gastric carcinoma. Oncol Rep 25: 989-995, 2011.

7. Kobayashi T, Sakaguchi M, Tanimoto R, Abarzua F, Takaishi M, Kaku H, Kataoka K, Saika T, Nasu Y, Miyazaki M, Kumon H and Huh NH: Mechanistic analysis of resistance to REIC/Dkk-3induced apoptosis in human bladder cancer cells. Acta Med Okayama 62: 393-401, 2008.

8. Wolter KG, Hsu YT, Smith CL, Nechushtan A, Xi XG and Youle RJ: Movement of Bax from the cytosol to mitochondria during apoptosis. J Cell Biol 139: 1281-1292, 1997.

9. De Giorgi F, Lartigue L, Bauer MK, Schubert A, Grimm S, Hanson GT, Remington SJ, Youle RJ and Ichas F: The permeability transition pore signals apoptosis by directing Bax translocation and multimerization. FASEB J: 16: 607-609, 2002.

10. Huang Z: Bcl-2 family proteins as targets for anticancer drug design. Oncogene 19: 6627-6631, 2000.

11. Van Laar VS and Berman SB: Mitochondrial dynamics in Parkinson's disease. Exp Neurol 218: 247-256, 2009.

12. Haque ME, Thomas KJ, D'Souza C, Callaghan S, Kitada T, Slack RS, Fraser P, Cookson MR, Tandon A and Park DS Cytoplasmic Pink1 activity protects neurons from dopaminergic neurotoxin MPTP. Proc Natl Acad Sci USA 105: 1716-1721, 2008.

13. Deng H, Jankovic J, Guo Y, Xie W and Le W: Small interfering RNA targeting the PINK1 induces apoptosis in dopaminergic cells SH-SY5Y. Biochem Biophys Res Commun 337: 1133-1138, 2005.

14. Nakajima A, Kataoka K, Hong M, Sakaguchi M and Huh NH: BRPK, a novel protein kinase showing increased expression in mouse cancer cell lines with higher metastatic potential. Cancer Lett 201: 195-201, 2003.
15. Murata H, Sakaguchi M, Jin Y, Sakaguchi Y, Futami J, Yamada H, Kataoka K and Huh NH: A new cytosolic pathway from a Parkinson disease-associated kinase, BRPK/PINK1: activation of AKT via mTORC2. J Biol Chem 286: 7182-7189, 2011.

16. Murata H, Sakaguchi M, Kataoka K and Huh NH: Multiple functions of PINK1 at different intracellular locations: beyond neurodegenerative diseases. Cell Cycle 10: 1518-1519, 2011.

17. Martin SA, Hewish M, Sims D, Lord CJ and Ashworth A: Parallel high-throughput RNA interference screens identify PINK1 as a potential therapeutic target for the treatment of DNA mismatch repair-deficient cancers. Cancer Res 71: 1836-1848, 2011.

18. Kimiya K, Naito S, Soejima T, Sakamoto N, Kotoh S, Kumazawa $\mathbf{J}$ and Tsuruo T: Establishment and characterization of doxorubicin-resistant human bladder cancer cell line, KK47/ ADM. J Urol 148: 441-445, 1992.

19. Masood A, Azmi AS and Mohammad RM: Small molecule inhibitors of Bcl-2 family proteins for pancreatic cancer therapy. Cancers 3: 1527-1549, 2011

20. Tanimoto R, Sakaguchi M, Abarzua F, Kataoka K, Kurose K, Murata H, Nasu Y, Kumon H and Huh NH: Down-regulation of BiP/GRP78 sensitizes resistant prostate cancer cells to genetherapeutic overexpression of REIC/Dkk-3. Int J Cancer 126: $1562-1569,2010$

21. Pizzo P and Pozzan T: Mitochondria-endoplasmic reticulum choreography: structure and signaling dynamics. Trends Cell Biol 17: 511-517, 2007.

22. Iwasawa R, Mahul-Mellier AL, Datler C, Pazarentzos E and Grimm S: Fis1 and Bap31 bridge the mitochondria-ER interface to establish a platform for apoptosis induction. EMBO J 30: 556-568, 2011.

23. Sakaguchi M, Kataoka K, Abarzua F, Tanimoto R, Watanabe M, Murata H, Than SS, Kurose K, Kashiwakura Y, Ochiai K, Nasu Y, Kumon H and Huh NH: Overexpression of REIC/Dkk-3 in normal fibroblasts suppresses tumor growth induction of interleukin-7. J Biol Chem 284: 14236-14244, 2009. 